\title{
Overview of the light-duty vehicles tailpipe emissions regulations in the European Union: status and upcoming type-approval and market surveillance schema
}

\begin{abstract}
In order to curb pollutant emissions from light-duty vehicles in the European Union, a set of complex regulations have been approved in the recent years (2016-2018) including more stringent emissions tests, independent in-service conformity checks, and a novel type-approval framework which includes market surveillance provisions to complement the type-approval requirements. Tailpipe emissions will need to meet stringent emission limits before entering the market at type-approval and at the end of the production line, as well as during their normal life under normal conditions of use. This contribution aims at providing a comprehensive but synthetic analysis of the current regulatory context in the EU.
\end{abstract}

Key words: WLTP, RDE, Market Surveillance, type-approval, in-service conformity

\section{Introduction}

Air pollution lies amongst the most significant risks in the European Union (EU) causing circa half a million premature deaths per year as well as significant economic and environmental impacts $[1,2]$. Road transport remains a key source of primary pollutants despite achieving substantial emissions reductions over the past decades $[1,3]$.

In order to further reduce tailpipe emissions from lightduty vehicles (LDVs) several policy instruments have been recently introduced in the EU. The Joint Research Center (JRC), the in-house research center of the European Commission has provided scientific and technical support to the development and implementation of the new laboratorybased test protocol, the WLTP as well as the on-road emissions test, the RDE.

The objective of the presentation is to provide insight on the current regulatory status in the EU regarding tailpipe emissions from LDVs in the laboratory and on the road, both at type-approval (i.e., homologation of emissions on a prototype vehicle), at the end of the production line, and during their real-life operation. In addition, an overview of the novel type-approval framework that will enter into force in 2020 is provided focusing on the role that has been assigned to JRC.

\section{Worldwide harmonised Light-duty vehicles Test Procedures (WLTP)}

The Commission Regulation (EU) $2017 / 1151$ of $1^{\text {st }}$ June 2017, implemented the WLTP into the EU legislation. The WLTP, in force in the EU since September $1^{\text {st }} 2017$, was first developed at the United Nations Economic Cooperation for Europe (UNECE) level [4]. The WLTP replaced the NEDC procedure as type-approval framework in the EU as the latter was outdated and did not adequately reflect real world emissions [5, 6]. Under the WLTP, the tailpipe emissions of LDVs (gases and particles), measured in laboratory conditions under standardized ambient conditions following the Type 1 test procedures (Annex XXI to regulation 2017/1151), shall meet the Euro 6 tailpipe emission limits set by the Regulation (EC) No 715/2007 and its amendments.

LDVs are driven on a chassis dynamometer on the Worldwide Light-duty Test Cycle (WLTC, Sub-Annex 1 to
Annex XXI), which is longer, more transient, and reaches higher vehicle speed than its predecessor the NEDC. The WLTP describes precisely how to determine the road load coefficients of a vehicle and how to transfer them to the chassis dynamometer (Sub-Annex 4), which was one of the main loopholes of the NEDC [7]. The WLTP, which allows road load determination on a wind tunnel or a test track, clearly defines important details like how to select the tires and which tire pressure and tread depth are permissible to perform the coast down test.

Vehicle preconditioning, soaking conditions, test cell temperature and humidity ranges, as well as the operation of sampling methods are comprehensively described in the regulation to limit the exploitable test flexibilities and enhance replicability and reproducibility of emission results (Sub-Annex 6). In particular, the WLTP prescribes a precise methodology to determine the gearshift points for manual transmission vehicles (Sub-Annex 2).

Specifications of the testing equipment are also widely covered in the WLTP (Sub-Annex 5), including the calibration, sampling frequency, accuracy, and response time requirements. According to the WLTP, the measurement principles for gaseous emissions are non-dispersive infrared (NDIR) absorption for carbon dioxide and carbon monoxide, flame ionization (FID) for hydrocarbons, and chemiluminescent (CLA) or non-dispersive ultra-violet resonance absorption (NDUV) for nitrogen oxides. Particulate matter mass is sampled on a single filter mounted within a holder in the sampled dilute exhaust gas flow and weighted in a temperature and humidity controlled weighing chamber. Fluorocarbon coated glass fibre filters or fluorocarbon membrane filters shall be used. The particle number sampling system shall consist of a volatile particle remover (VPR) upstream of a particle number counter (PNC) and suitable transfer tubing.

The WLTP also describes the testing conditions and tailpipe emission limits applying for positive ignition vehicles at idling (Type 2 test: limiting $\mathrm{CO}$ emissions at normal and high engine idle speeds) and at low ambient temperatures (Type 6 test: covering $\mathrm{CO}$ and hydrocarbons emissions at cold start at $-7^{\circ} \mathrm{C}$ ). The protocols for verifying the durability of pollution control devices (Type 5 test) lie also under the WLTP umbrella. 
The Conformity of Production (CoP) is an additional check on emissions compliance performed by the manufacturer on vehicles at the end of the production line. At least one verification per 5000 vehicles produced or once per year, whichever comes first, shall be performed on each CoP vehicle family (vehicles within the same family have the same fuel type, combustion type, engine displacement, transmission type, etc.). The vehicles shall conform to the Euro 6 emission limits according to a precise statistical procedure and testing protocol, which considers the emission results of a set of three up to sixteen vehicles (Fig. 1). The Type Approval Authority (TAA) shall select randomly the vehicles until a pass or fail decision is reached according to the statistical procedure. It is responsibility of the TAA to audit the CoP documentation at least once per year and to perform additional physical tests in case it is not satisfied with the auditing procedure or at least once every three years.

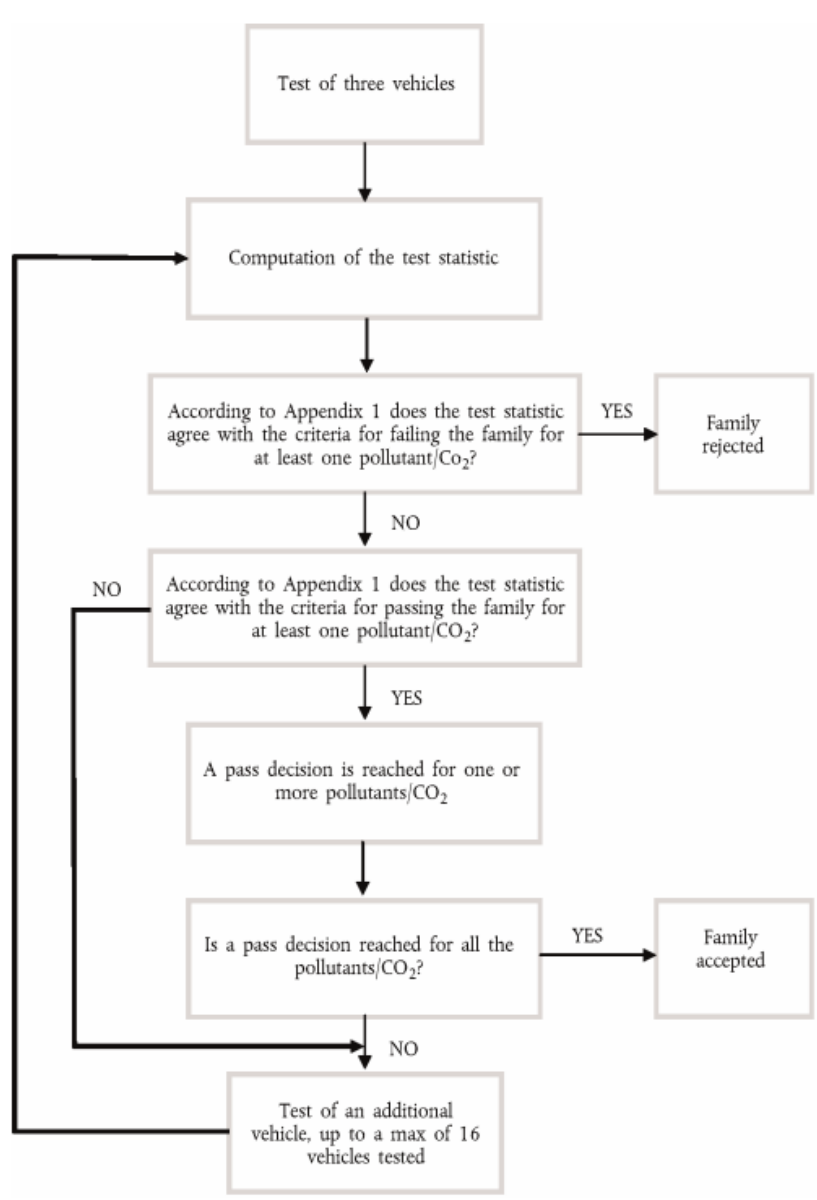

Fig. 1. Overview of the procedure to check the conformity of the vehicle for a Type 1 test [1]

\section{Real Driving Emissions (RDE) regulation}

Following the approach used to assess the emissions performance of heavy-duty vehicles under real life operation through Portable Emissions Measurement Systems (PEMS), the EU has developed the Real Driving Emissions (RDE) regulation for LDVs in the period 2011-2018. The RDE aims at securing low tailpipe emissions of passenger cars and light commercial vehicles under real traffic operation under normal conditions of use.
The RDE, which has been introduced in the EU legislation through four regulatory packages (RDE1: EU 2016/ 427, RDE2: EU 2016/646, RDE3: EU 2017/1154, and RDE4: EU 2018/1832), is applicable for all new vehicles since September $1^{\text {st }}$ 2018. Vehicles tested under the RDE regulation need not-to-exceed (NTE) certain nitrogen oxides and solid particle number limits over a route that complies with a series of testing boundaries (ambient temperature between $-7^{\circ} \mathrm{C}$ and $35^{\circ} \mathrm{C}$, maximum altitude of 1300 m.a.s.l., test duration between 90 and 120 minutes, at least $16 \mathrm{~km}$ driven on urban, rural and motorway conditions, etc.). The RDE boundary conditions were set in a way to cover most of the driving situations within the EU while allowing PEMS to be used within their optimal operating conditions. The fact that on-road tests complying with the $\mathrm{RDE}$ requirements can be performed in countless combinations of ambient and traffic conditions, driving styles, and payload largely limits the potential of cycle optimization (as occurred in the past when vehicle emissions were checked only under the NEDC test protocol). The fact that RDE NTE limits need to be met under a wide range of conditions has become a major driver of current engine and after-treatment systems development over the last years [8].

RDE1 describes the testing procedure, provides the technical requirements for PEMS and defines the ex-post emissions calculations and evaluation methods. RDE2 introduced the NTE compliance request to the urban driving on top of the entire RDE trip and set new boundary conditions for diving dynamics and cumulative positive elevation gain. The extended documentation package, which consists of a description of the base and auxiliary emissions strategies to be shared with the TAA was introduced in RDE2 as a way to prevent defeat devices. RDE3 comprised a provision to include emissions during cold start operation in the final RDE emission values, and set the technical parameters to assess emissions of plug-in hybrid electric vehicles. RDE4 introduced the In-Service Conformity (ISC) provisions to evaluate the emissions performance of vehicles during their normal life as well as modifications to the ex-post evaluation methods to ensure practicality and effective emissions testing.

The NTE limit is the Type I test limit multiplied by a conformity factor $(\mathrm{CF})$ that includes a margin accounting for the additional measurement uncertainty of PEMS relative to standard laboratory equipment. $\mathrm{NO}_{\mathrm{x}} \mathrm{CF}$ was set by RDE2 and modified in RDE4, whereas the PN CF was introduced in RDE3. The RDE regulation includes a procedure to review the margin in light of technological improvements of PEMS. The JRC has developed a methodological framework for the revision of the $\mathrm{NO}_{\mathrm{x}}$ margin [9] that uses the error propagation rule and takes into account the uncertainties of gas analysers, exhaust mass flowmeters, and global positioning system. The $\mathrm{NO}_{\mathrm{x}}$ margin applicable to new type approved vehicles from January 2020 was set at 0.43 considering a worst-case scenario for zero $\mathrm{NO}_{\mathrm{x}}$ drift.

In order to support best practices and avoid wrongdoings during on-road emissions tests in the context of the $\mathrm{RDE}$ regulation, the JRC has developed a guidance document [10] covering all aspects of PEMS testing from installation (Fig. 2) to calibration and execution of tests. 


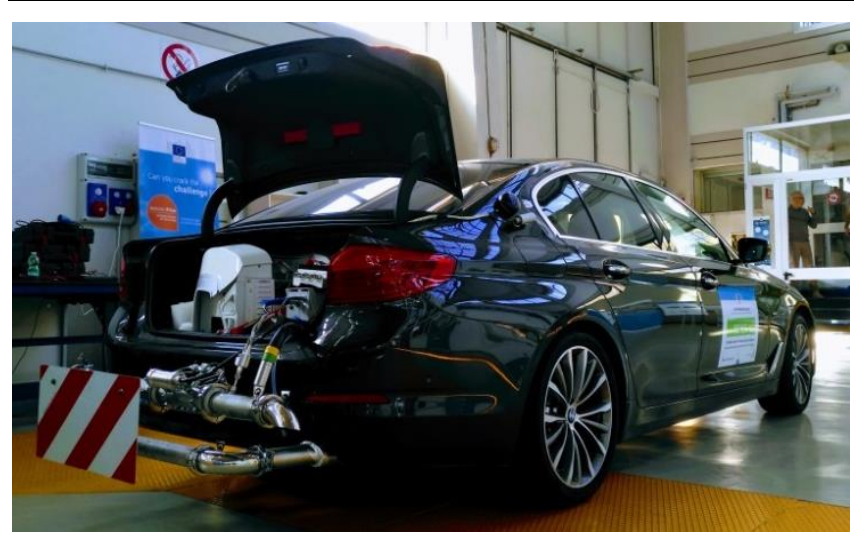

Fig. 2. PEMS installation [2]

\section{In-Service Conformity (ISC)}

From September $1^{\text {st }} 2019$, the ISC provisions will be applicable to all new vehicles to ensure that LDVs comply with their emission limits throughout their normal life (up to a mileage of $100,000 \mathrm{~km}$ or five years, whichever occurs first) and not only at type-approval. ISC, which was introduced by EU regulation 2018/1832 of November $5^{\text {th }} 2018$ (RDE4), defines the procedures and responsibilities to check tailpipe emissions compliance during RDE as well as WLTP Type 1 and Type 6 tests.

In order to prioritize the ISC testing, the Granting TypeApproval Authorities (GTAA) will perform risk assessments based on remote-sensing (Fig. 3), simplified measurement systems and/or other sources of tailpipe emissions. The outcome of the risk assessment shall be a list of vehicle families with potential issues regarding their emissions performance that will undergo detailed scrutiny through actual ISC testing.
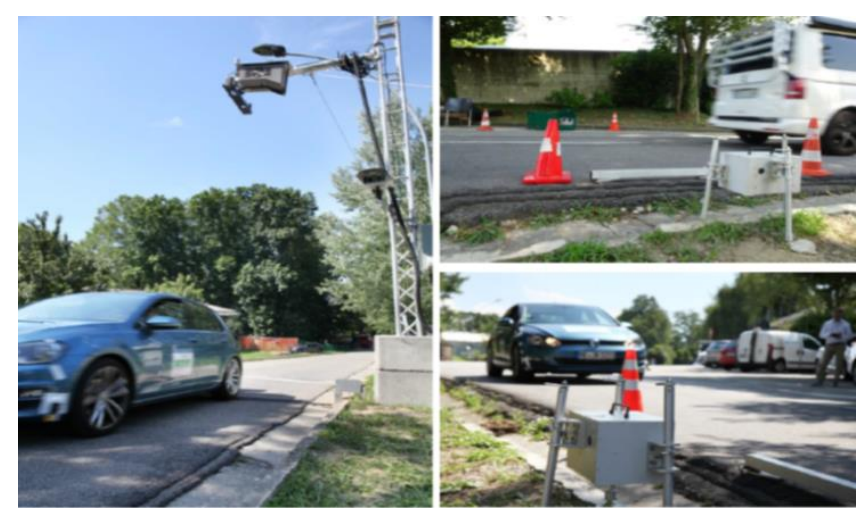

Fig. 3. Remote-sensing instruments are placed in the road-side and provide an instantaneous reading of vehicle emissions passing-by [3]

ISC tests shall be performed by vehicle manufacturers, GTAAs, and may be additionally performed by third parties through accredited laboratories. The accreditations seek to guarantee that vehicle inspection (ISO 17020) and emissions compliance checks (ISO 17025 and) are fair, properly executed, and that instrumentation fully complies with the regulation requirements. Accredited laboratories will have access to transparency lists, which contain the necessary information to perform testing in type-approval like conditions (road load settings, how to enable dyno mode, gearshifting pattern, etc.). Manufactures will need to perform
ISC testing at least on 5\% of their families every year (with a minimum of two families). In order to strengthen the ISC procedure, the GTAA is additionally responsible to audit the ISC testing performed by the manufacturer and accredited laboratories. The ISC procedure allows, for the first time in history, that consumer and environmental organizations, for example, officially confront the emissions compliance of vehicles through accredited laboratories.

The results of ISC tests will be pooled together into a publicly accessible electronic platform hosted by the European Commission (different user profiles will have different access to data privileges). An ad-hoc ISC statistical procedure has been developed to robustly assess the emissions compliance of a given vehicle family while minimising the testing burden, the environmental risk (compliance with ISC but incompliance with NTE limits), and the manufactures' risk (incompliance with ISC but compliance with NTE limits). For RDE and WLTP Type 1 tests, the ISC pass-or-fail decision requires a minimum of three vehicles and is reached with a maximum of ten vehicles depending on the number of individual failed tests (Fig. 4). A simplified statistical approach is used for WLTP Type 6 test where a decision may be reached after three tests.

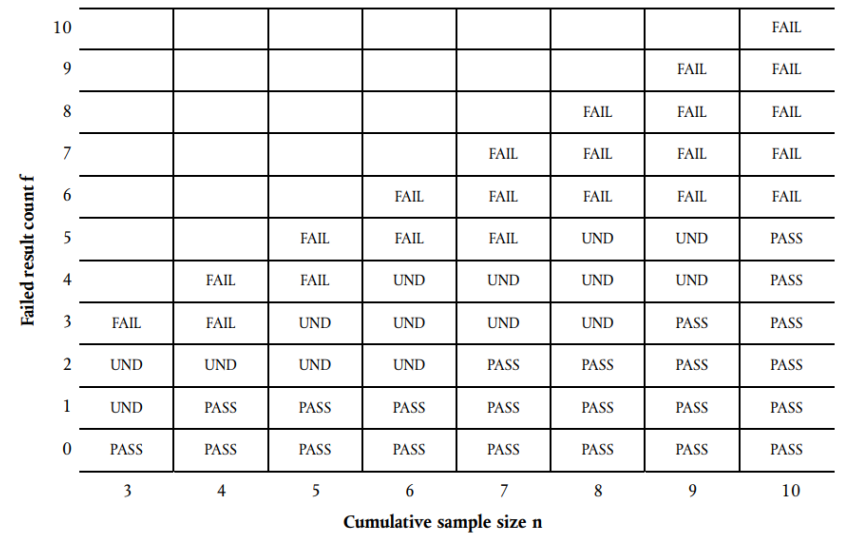

Fig. 4. Decision chart for the ISC statistical procedure applying for typeapproved vehicles from January $1^{\text {st }} 2020$ (UND: undecided) [4]

On top the statistical procedure described above, an additional clause is defined for outliers, i.e., vehicles that emit pollutants way above the NTE limits. The presence of outliers in ISC tests may lead to a direct fail outcome for the whole family even if the minimum number of tests is not yet reached. The presence of two intermediate outliers (emissions $>1.3 *$ NTE limit) or one extreme outlier (emissions $>2.5 *$ NTE limit) in a sample shall lead to a fail of the sample.

Within 10 days of the end of the ISC testing, the GTAA shall start detailed investigations with the manufacturer in order to decide whether the ISC family passes or fails the ISC procedure. In case of excess emissions, the manufacturer shall provide to the GTAA a description of the "possible cause of the failures, which parts of the family might be affected, whether other families might be affected, or why the problem which caused the failure at the original ISC tests is not related to in-service conformity, if applicable'. In the case a vehicle family does not comply with the emissions limits comprehensive remedial measures shall be put 
in place by the vehicle manufacturer to restore effective and durable after-treatment systems. The plan describing the remedial measures shall be provided to the GTAA within 45 working days including a demonstration of the effectiveness and durability of the proposed measures and a communication plan to vehicle owners and repair workshops. Within 15 working days, the GTAA needs to accept or decline the remedial plan. If the GTAA does not accept it, it is entitled to take all appropriate measures to restore conformity, including withdrawal of the type-approval and notify its decision to all Member States and the Commission. Member States shall take measures to ensure that the approved plan of remedial measures is applied within two years to at least $90 \%$ of affected vehicles registered in their territory.

In order to foster transparency, the ISC regulation foresees that the GTAA needs to make publicly available and free of charge, a report containing all the results of all the finalised ISC investigations of the previous year, by the 31 March of each year.

The JRC is currently working on accrediting its testing procedures for RDE and WLTP (ISO 17025) and for vehicle inspection (ISO 17020) in order to be in the position to actively participate in ISC testing.

\section{New Type-Approval Framework and Market Surveillance}

EU regulation 2018/858 of May $30^{\text {th }} 2018$ describes the new framework for the type-approval and CoP of motor vehicles in the EU that will replace Directive 2007/46/EC from September $1^{\text {st }} 2020$. As overall goals, the new framework pursues raising the technical quality and robustness of the type-approval procedure, facilitate its enforcement, prevent market distortions, increase transparency, minimize the administrative burden, and foster the independence of authorities in order to ensure a high level of safety and environmental performance of light and heavy-duty vehicles.

The new framework entitles Member States to act against non-compliant vehicles on their territory without the need to wait for the GTAA to take action.

In order to strengthen the collaboration among Member States, the regulation foresees the creation of an advisory Forum for Exchange of Information on Enforcement that shall include representatives of national authorities and the Commission.

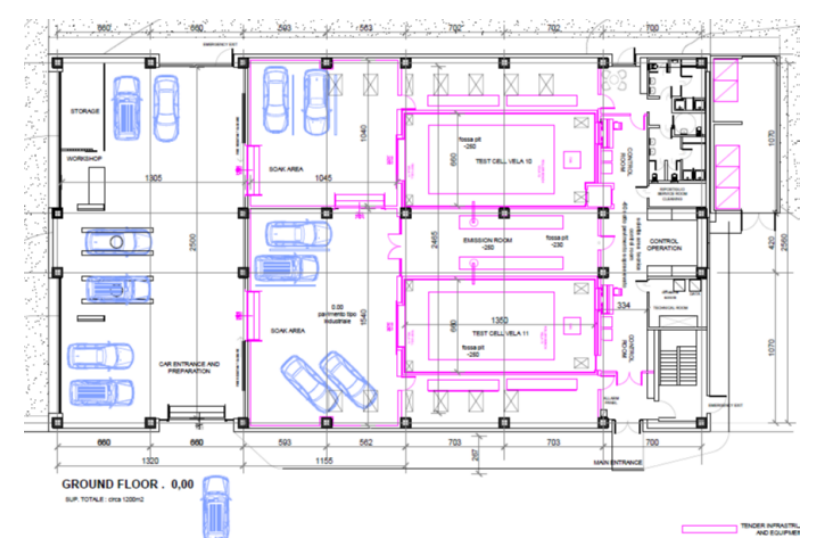

Fig. 5. New Vehicle Emissions Laboratories (VELA 10 and VELA 11) building plan [5]
The new type-approval framework introduces also an effective market surveillance system to control the conformity of vehicles already in circulation. In this sense, Member States as well as the European Commission will name Market Surveillance Authorities responsible to perform independent vehicle compliance tests on vehicles already on the market. The European Commission oversight of the whole type-approval procedure includes taking measures (e.g., ordering vehicle recalls, giving fines, and even revoking type-approval certificates) against noncompliant vehicles. The Commission shall also audit the procedures put in place by approval authorities to grant type-approvals, and execute conformity of production at least once every five years.

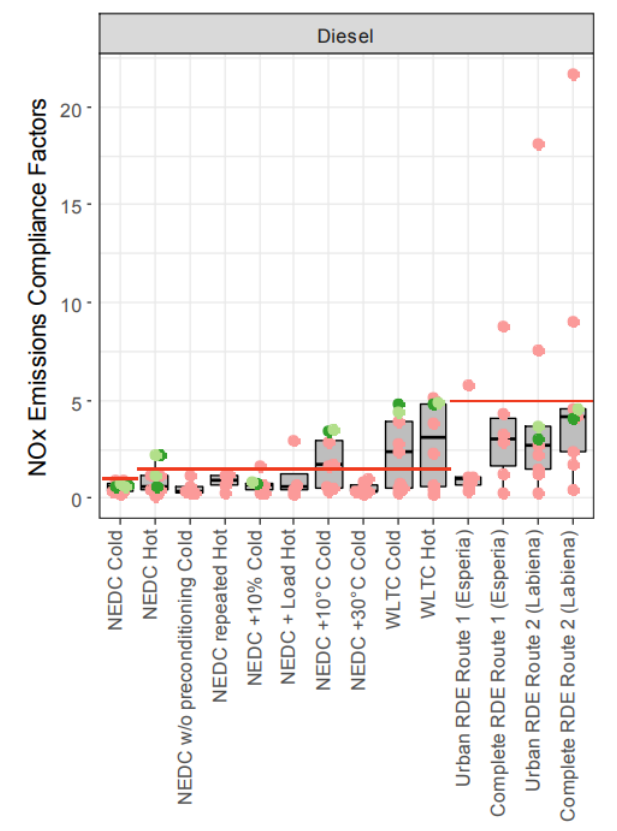

Fig. 6. $\mathrm{NO}_{\mathrm{x}}$ emissions $/ \mathrm{NO}_{\mathrm{x}}$ standards (Compliance Factors) over laboratory and on-road tests. Horizontal red lines indicate limits or recommended thresholds for Compliance Factors. Green and red dots represent individual Euro 5b and Euro 6b vehicles, respectively [6]

The JRC will be the European Commission Service responsible for the execution of the market surveillance tests (emissions and safety) as well as inspection of the work of technical services (peer-review mechanism). New personnel, facilities (Fig. 5), and instrumentation have been allocated at JRC to start carrying out Market Surveillance activities from September $1^{\text {st }} 2020$.

Since early 2017, the JRC has been carrying out independent tailpipe emissions compliance checks on LDVs in the laboratory and on the road, following standard and alternative NEDC, WLTP and RDE procedures [11]. The alternative laboratory tests include back-to-back NEDC and WLTP tests (i.e., with warm engine), setting the test cell at $10^{\circ} \mathrm{C}$ and $30^{\circ} \mathrm{C}$, modifying the speed trace, etc. The outcomes of such activities aim at contributing to the development of robust vehicle screening methodologies and testing protocols in the context of upcoming Market Surveillance obligations.

The work conducted at JRC also focus on developing and enhancing testing protocols and data processing tools to 
identify vehicles that present emission patterns which could be caused by potential use of defeat devices (Fig. 6).
Acknowledgements

This work is presented on behalf of the colleagues of the Sustainable Transport Unit (JRC) supporting the development and implementation of vehicle emissions regulations in the EU.

\section{Nomenclature}

$\mathrm{CF} \quad$ conformity factor

CoP conformity of production

GTAA Granting Type-Approval Authority

ISC In-Service Conformity

JRC Joint Research Center

NEDC New European Driving Cycle
PEMS Portable Emissions Measurement System

RDE Real Driving Emissions

TAA Type Approval Authority

WLTP Worldwide harmonized Light-duty vehicles Test Procedures

\section{Bibliography}

[1] EUROPEAN ENVIRONMENT AGENCY. EEA Report No 12/2018 Air quality in Europe - 2018 report https://doi.org/10.2800/777411

[2] CE DELFT. Health impacts and costs of diesel emissions in the EU. 2018, Publication code: 18.4R30.140.

[3] EUROPEAN ENVIRONMENT AGENCY. EEA Briefing No 15/2018. Progress of EU transport sector towards its environment and climate objectives. https://doi.org/10.2800/954310

[4] TUTUIANU, M., CIUFFO, B., HANIU, T. et al. Development of a world-wide harmonized light duty test cycle (WLTC). Transportation Research Part D: Transport and Environment. 2015, 40, 61-75.

https://doi.org/10.1016/j.trd.2015.07.011

[5] FONTARAS, G., ZACHAROF, N., CIUFFO, B. Fuel consumption and $\mathrm{CO}_{2}$ emissions from passenger cars in Europe Laboratory versus real-world emissions. Progress in Energy and Combustion Science. 2017, 60, 97-131.

https://doi.org/10.1016/j.pecs.2016.12.004

[6] WEISS, M., BONNEL, P., HUMMEL, R. et al. On-road emissions of light-duty vehicles in Europe. Environmental Science \& Technology. 2011, 45, 8575-8581.

https://doi.org/10.1021/es2008424
[7] PAVLOVIC, P., CIUFFO, B., FONTARAS, G. et al. Assessment of test cycle flexibilities, driving behavior, and their impacts on $\mathrm{CO}_{2}$ results. TRB $96^{\text {th }}$ Annual Meeting. 2017.

[8] BIELACZYC, P., WOODBURN, J., Trends in automotive emission legislation: impact on LD engine development, fuels, lubricants and test methods: a global view, with a focus on WLTP and RDE regulations. Emission Control Science and Technology. 2019, 5, 86-98. https://doi.org/10.1007/s40825-019-0112-3

[9] GIECHASKIEL, B., CLAIROTTE, M., VALVERDE, V. et al. Framework for the assessment of PEMS (Portable Emissions Measurement Systems) uncertainty. Environmental Research. 2018, 166, 251-260. https://doi.org/10.1016/j.envres.2018.06.012

[10] VALVERDE, V., BONNEL, P. On-road testing with Portable Emissions Measurement Systems (PEMS). Guidance note for light-duty vehicles. JRC Technical Report. 2018. https://doi.org/10.2760/08294

[11] ClairotTe, M., VAlVerde, V., BONNEL, P. et al. Joint Research Centre 2017 light-duty vehicles emissions testing. Contribution to the EU market surveillance: testing protocols and vehicle emissions performance. 2018. https://doi.org/10.2760/83327

Víctor Valverde-Morales, Ph.D. - Sustainable Transport Unit Joint Research Center, European Commission.

e-mail: victor.valverde-morales@ec.europa.eu 